\title{
La valorisation économique des forêts : entre filière et territoire
}

The economic valorization of forests: between commodity chain and territory

Philippe Méral, Vahinala Raharinirina, Fano Andriamahefazafy et Djohary Andrianambinina

\section{(2) OpenEdition}

1 Journals

Édition électronique

URL : https://journals.openedition.org/economierurale/936

DOI : $10.4000 /$ economierurale.936

ISSN : 2105-2581

Éditeur

Société Française d'Économie Rurale (SFER)

Édition imprimée

Date de publication : 1 novembre 2006

Pagination : 74-89

ISSN : 0013-0559

\section{Référence électronique}

Philippe Méral, Vahinala Raharinirina, Fano Andriamahefazafy et Djohary Andrianambinina, « La

valorisation économique des forêts : entre filière et territoire ", Économie rurale [En ligne], 294-295

Juillet-octobre 2006, mis en ligne le 23 octobre 2009, consulté le 21 septembre 2021. URL : http://

journals.openedition.org/economierurale/936 ; DOI : https://doi.org/10.4000/economierurale.936

(C) Tous droits réservés 


\section{La valorisation économique des forêts : entre filière et territoire}

Philippe MÉRAL • Chargé de recherches IRD, membre de I'UMR M 063 C3ED (Centre d'Economie et d'Éthique pour l'Environnement et le Développement), Institut de Recherche pour le Développement et Université de Versailles Saint-Quentin-en-Yvelines

Vahinala RAHARINIRINA • Doctorante, membre de I'UMR M 063 C3ED (Centre d'Economie et d'Éthique pour l'Environnement et le Développement), Institut de Recherche pour le Développement et Université de Versailles Saint-Quentin-en-Yvelines

Fano ANDRIAMAHEFAZAFY • Doctorant, membre de I'UMR M 063 C3ED (Centre d'Economie et $d^{\prime} E$ thique pour l'Environnement et le Développement), Institut de Recherche pour le Développement et Université de Versailles Saint-Quentin-en-Yvelines et membre du C3EDM (Centre d'Economie et d'Éthique pour l'Environnement et le Développement à Madagascar), Université d'Antananarivo.

Djohary ANDRIANAMBININA • Doctorant, membre de I'UMR M 063 C3ED (Centre d'Economie et d'Ethique pour l'Environnement et le Développement), Institut de Recherche pour le Développement et Université de Versailles Saint-Quentin-en-Yvelines et membre du C3EDM (Centre d'Economie et d'Éthique pour l'Environnement et le Développement à Madagascar), Université d'Antananarivo.

$\mathbf{L}$ e développement de filières permettant la valorisation économique des forêts (produits forestiers non ligneux et services écotouristiques) fait l'objet d'une attention particulière tant par les bailleurs de fonds intervenant dans les pays en développement qu'au sein des milieux académiques. Il s'agit, dans une perspective de développement durable, de mettre en valeur à travers le développement d'une filière marchande certaines ressources naturelles ou certains services environnementaux fournis par des écosystèmes menacés de dégradation anthropique. Ces actions sont justifiées par la recherche d'alternatives économiques sur lesquelles peuvent s'appuyer les populations locales afin de limiter la pression sur ces écosystèmes.

Comme le soulignent Belcher et RuizPérez (2001) ${ }^{1}$, les expériences se sont multipliées depuis une quinzaine d'années, si bien que, même si tous s'accordent à considérer que les revenus de la valorisation sont très faibles par rapport à ceux

\footnotetext{
1. Belcher B. Ruiz-Pérez M. An International Comparison of Cases of Forest Product Development: Overview, Description and Data Requirements. Center for International Forestry Research, Working Paper, 2001, 24 p.
}

issus des pratiques impliquant la déforestation (exploitation non durable du bois d'œuvre, conversion des forêts en parcelle agricole...), il est possible de trouver des exemples décevants et d'autres plus prometteurs. De ce constat mitigé sont nées deux tendances (Wunder, 2005) ${ }^{2}$. Pour certains, la lenteur avec laquelle ces activités permettent de limiter les pressions sur les forêts justifie aujourd'hui le recours à des pratiques plus directes de paiements des services environnementaux (contrat de conservation par exemple). Pour d'autres, au contraire, la promotion de ces filières de valorisation devrait permettre un développement local endogène plus important que par les paiements directs. Au lieu de les délaisser, il convient plutôt d'identifier les mécanismes permettant de rendre effectives et durables ces activités de valorisation.

La thèse que nous soutenons ici, dans l'esprit de ce deuxième courant d'analyse, est que cette effectivité et surtout sa dura-

2. Wunder S. Payments for Environmental Services: some nuts and boots. Cifor, Occasional Paper, $\mathrm{n}^{\circ}$ 42, 32 p. disponible sur http:// www.cifor.cgiar.org/publications/pdf_files/occ Papers/OP-42.pdf 
bilité dépend en grande partie de l'orthogonalité de ces filières de valorisation ; c'est-à-dire de la prise en compte au sein des filières (axe vertical) de la dimension territoriale (axe horizontal) concernant autant les acteurs impliqués dans la valorisation que les actifs ainsi valorisés (Requier-Desjardins, 2005). De manière implicite, ceci signifie que la préférence pour telle ou telle option ne doit pas dépendre d'une seule comparaison des bénéfices économiques perçus (ou à percevoir si compensation réelle) par les populations mais aussi et surtout de leur effet structurant au niveau local ; ce qui implique une réflexion plus élaborée qu'une seule évaluation économique de type coûts-avantages.

Ces enjeux autour de l'effectivité des filières de valorisation sont d'autant plus importants que la plupart des parties prenantes des politiques environnementales au sein des pays en développement cherchent à promouvoir ce type d'action. Ainsi, à Madagascar, la valorisation de la biodiversité fait l'objet d'un soutien explicite à travers plusieurs dispositifs à la fois institutionnels comme la mise en place d'une unité de soutien à la valorisation au sein de l'Office national de l'environnement en 1996 ou, plus récemment, à travers la mise en œuvre de la troisième phase du plan environnemental qui met l'attention sur «l'adhésion des populations locales à des projets de valorisation économique de leur biodiversité » et opérationnels à travers le financement par les bailleurs de fonds de projets de valorisation de la biodiversité (appui aux groupements paysans, structuration de la filière...).

Dans ce contexte tant théorique que pratique, cet article se fixe pour objectif de discuter de ces contraintes en prenant appui non pas sur un seul actif mais sur un territoire particulier, en l'occurrence la forêt des Mikea dans le sud-ouest malgache. La logique d'intervention des bailleurs de fonds et de leurs relais associatifs locaux est de mettre l'accent sur une combinaison de plu- sieurs activités de valorisation au sein d'un territoire donné.

Une première partie est consacrée à la description du site d'étude et aux enjeux de cette valorisation dans une forêt marquée par une forte déforestation et par un activisme institutionnel très prononcé. Fort de ce constat, nous portons notre attention, dans une deuxième partie, sur les trois filières promues par les institutions : l'apiculture, les plantes médicinales et l'écotourisme. Le bilan très mitigé de cette valorisation nous conduit alors à identifier les contraintes communes à ces différentes expériences qui deviennent alors autant de conditions nécessaires pour rendre effectives ces activités de valorisation.

\section{Déforestation et action collective}

La forêt des Mikea se trouve dans le sudouest de Madagascar, à $40 \mathrm{~km}$ au nord de Toliara, capitale de la province du même nom $^{3}$. Cette forêt dense sèche subit des pressions anthropiques depuis de nombreuses années en relation avec les activités de culture de rente (pois du cap, coton...) développées dans le couloir d'Antseva (Fiéloux et Lombard, 1990). Au milieu des années 1990, le rythme de la déforestation s'est fortement accéléré (Razanaka et al. 2001).

\section{La dynamique économique de la déforestation}

Les causes de la déforestation sont nombreuses, même si la principale est liée à la culture du maïs sur abattis-brûlis. En raison du faible respect des autorisations d'occupation de la forêt qui relèvent de l'administration des Eaux et Forêts, les agriculteurs, des masikoro rejoints par de nombreux migrants attirés par des profits rapides et importants, obtenus sans équipement et

3. Plus précisément, la forêt des Mikea s'étend entre les latitudes $22^{\circ} 15^{\prime} \mathrm{S}$ et $23^{\circ} 00 \mathrm{~S}$. Elle est délimitée par le canal du Mozambique à l'ouest et par la RN 9 à l'est. 
presque sans main-d'œuvre, utilisent l'espace forestier afin d'y cultiver du maïs (Blanc-Pamard et al, 2005).

L'origine du boom du maïs est liée à la mise en place du programme Poséidom de l'Union européenne qui a permis à l'Île de la Réunion de développer son activité porcine et de susciter de nouveaux marchés d'approvisionnement en maïs pour l'alimentation. De par sa proximité, Tuléar est devenue rapidement un fournisseur d'appoint des coopératives réunionnaises (Fauroux S., 2000). Cette filière relativement courte, puisque seuls deux à trois intermédiaires permettent de relier le producteur de maïs aux éleveurs porcins de la Réunion, est dominée par l'aval selon des variables échappant totalement aux paysans malgaches (différentiel de prix d'achat du maïs entre l'Argentine et Madagascar et la capacité de stockage à la Réunion, essentiellement) qui produisent de surcroît un actif parfaitement générique (Minten et Méral, 2006).

La culture du maïs est réalisée en pratiquant la transformation de la forêt en terre agricole. Les techniques culturales sont finalement classiques : les paysans combinent les facteurs de production travail et terre, en relative abondance, ce qui permet une culture extensive. En l'absence de capital technique, les rendements à l'hectare qui dépassent rarement les deux tonnes par an, diminuent dès la troisième année d'exploitation, rendant attractif la défriche de nouvelles terres. Seuls l'éloignement progressif des fronts pionniers par rapport aux villages (et surtout des points d'eau) et la contrainte sur la disponibilité en main-d'œuvre freinent naturellement le processus (Aubry et Ramaromisy, 2003 ; Aknin et al, 2007). Cette rentabilité de la culture sur défriche-brûlis, pratique qui est une des principales causes de la déforestation de par le monde, montre l'ampleur des actions de lutte contre la déforestation et notamment les conditions nécessaires pour que des alternatives économiques soient attractives.

\section{Action collective}

Pour faire face à cette déforestation, plusieurs initiatives ont été prises depuis 1996, date de la création de l'association Fimami (Fikambanana Miaro ny Alan'ny Mikea que l'on peut traduire par Association pour la Protection de la Forêt des Mikea), autour de laquelle se sont structurées l'ensemble des actions de lutte contre la déforestation et de valorisation économique (Méral et Raharinirina-Douguet, 2006). Si peu de choses sont réalisées jusqu'en 2000, la Fimami a profité de cette période pour institutionnaliser son rôle :

(i) en tant que partenaire des agences d'exécution de la politique environnementale et des ONG tels que le WWF,

(ii) en tant qu'acteur de la nouvelle politique de décentralisation, en devenant une structure intercommunale de concertation,

(iii) en tant qu'acteur de la nouvelle politique de développement rural mise en œuvre en 1999.

Un des points communs à ces trois politiques et aux institutions en charge de les mettre en œuvre est de s'appuyer sur un processus participatif de planification ascendante. L'idée est de faire ressortir les priorités de développement et de gestion durable du terroir par la population locale (d'abord au niveau du quartier puis de la commune et enfin au niveau intercommunal sous l'égide de la Fimami) et de les structurer dans des documents susceptibles de servir de base de discussion avec les bailleurs de fonds (Andriananja et Raharinirina 2004 ; Froger et al. 2004). Même si les projets sont parfois insufflés par les institutions et les maires qui ont une connaissance fine des opportunités de financement, ils apparaissent comme le produit d'une volonté des communautés locales et, à ce titre, bénéficient d'une légitimité reconnue par l'ensemble des acteurs.

Parmi les projets de valorisation de la biodiversité, trois d'entre eux, l'apiculture, les plantes médicinales et le tourisme, bénéficient d'un contexte naturel propice et d'un 
soutien des institutions. Ainsi, la filière plantes médicinales est apparue dans les documents de planification en raison d'une forte biodiversité étudiée par diverses institutions scientifiques. Il en est de même pour l'apiculture qui bénéficie à la fois de l'abondance des essences mellifères dans la forêt et d'un soutien fort de la part de bailleurs de fonds dans des régions voisines. Enfin, la proximité de sites touristiques d'importance, Ifaty-Mangily, au sud de la forêt a également joué en faveur du choix de cette activité à valoriser.

\section{Des initiatives qui tardent à produire leurs effets}

Le constat général que nous avons fait à la suite des trois années passées dans cette zone d'étude est que malgré la volonté des différents acteurs de promouvoir ces activités de valorisation, celles-ci sont encore balbutiantes tant au niveau institutionnel (difficulté de fédérer les paysans en groupements...) qu'économiques (faiblesse des revenus). Parallèlement, les autorités ont mis en place des mesures drastiques de lutte contre la déforestation depuis 2002 ce qui a pour effet d'accroître la vulnérabilité des petits paysans (Aknin et al., 2007 ; Méral et Raharinirina-Douguet, 2006). Leurs conditions de vie se sont détériorées et les espoirs placés dans la mise en œuvre de projets alternatifs (remise en culture des terres abandonnées avec apport en capital technique, valorisation économique des produits forestiers non ligneux...) ont disparu. Les projets de mise en œuvre d'une aire protégée au sein de la forêt des Mikea maintiennent pour autant l'intérêt pour ces approches si bien qu'il apparaît essentiel encore aujourd'hui, et au-delà de l'intérêt scientifique, d'identifier les raisons pour lesquelles ces initiatives tardent à produire leurs effets.

\section{La filière plantes médicinales}

La forêt des Mikea abrite plusieurs types de plantes médicinales qui répondent à diffé- rents besoins de la population locale : usage thérapeutique, cultuel, spirituel, invocation d'esprit, etc. Par ailleurs, la pharmacopée constitue l'usage le plus important des ressources forestières de la forêt des Mikea : $29 \%$ pour la pharmacopée contre $23 \%$ pour l'habitat et $12 \%$ pour l'alimentation selon CNRE et al. (2001) ${ }^{4}$. À l'instar de nombreux pays d'Afrique, la pharmacopée occupe donc une place centrale pour la population locale d'autant que la pauvreté limite l'accès à la médecine moderne (Sunderland et Ndoye, 2004).

Pour le moment, la filière se distingue en deux segments : un très court et un autre moyen. La première forme est courte très localisée. Elle met en relation les tradi-praticiens qui disposent du savoir-faire, aux autres villageois souvent au sein même de la communauté. L'échange se fait la plupart du temps sous la forme de troc avec une transmission orale des prescriptions. L'activité du tradi-praticien est ponctuelle, souvent associée à une ou plusieurs autres activités. $\mathrm{La}$ cueillette des plantes se fait selon les besoins. La valeur d'échange n'est pas uniquement liée au type de plante mais aussi à la nature de la maladie, sa gravité, et la plupart du temps à la rapidité et l'efficacité des soins ; les services sont souvent rémunérés en nature.

La filière la plus longue concerne l'approvisionnement en plantes médicinales de la ville de Tuléar. Il existe un marché qui vend de manière régulière les plantes médicinales en provenance de la forêt. Dans ce cas, la structuration de la filière peut être qualifiée de classique avec les producteurs et les consommateurs mis en relation par des intermédiaires. Les revenus varient très fortement en fonction du statut de chaque acteur. En principe, ce sont les intermédiaires, c'est-à-dire ceux qui ont

4. CNRE, ONE, FIMAMI, CRP. Valorisation des filières potentielles en matière de biodiversité : les plantes médicinales de la région de la forêt des Mikea. Mimeo, 2001. 
Tableau 1. Projets apicoles dans la forêt des Mikea

\begin{tabular}{l|c|c|c}
\hline & Manombo & Ankililoaka & Analamisampy \\
\hline Institution de soutien & PSDR & PSDR & PSDR \\
& $2002-2003$ & $2002-2003$ & début 2004 \\
\hline Montant de l'aide & 40 millions FMG & 17 millions FMG & 35 millions FMG \\
(équivalent en euros) & $(3200 €)$ & $(1360 €)$ & $(2800 €)$ \\
\hline Nombre de ruches prévues & 200 & 160 & 200 \\
\hline Nombre de ruches habitées & $\begin{array}{c}10 \\
\text { septembre 2003 }\end{array}$ & $\begin{array}{c}28 \\
\text { septembre 2003 }\end{array}$ & $\begin{array}{c}\text { aucune } \\
\text { septembre 2004 }\end{array}$ \\
\hline
\end{tabular}

FMG : Franc malgache

Source : calculs des auteurs

accès au marché de l'agglomération de Tuléar qui font le maximum de gains ${ }^{5}$.

La forêt des Mikea est incontestablement riche en plantes médicinales de valeur économique et scientifique non négligeable. Mais contrairement à plusieurs régions de Madagascar, les institutions prônant la valorisation n'ont pas réussi à associer les opérateurs, nationaux ou étrangers (Raharinirina, 2004) ${ }^{6}$. Pour le moment, le bilan est donc négatif dans la mesure où la valorisation des plantes médicinales et aromatiques de la forêt des Mikea n'est encore qu'au stade des intentions.

\section{La filière apiculture}

La promotion de l'activité apicole se justifie de deux manières : d'une part en raison d'un nombre de projets similaires dans la plupart des régions malgaches et par conséquent d'une forme de savoir-faire en matière de valorisation (Lagarde et Rakotovelo, 2004) et, d'autre part, en raison de pratique traditionnelle au sein de la forêt des Mikea. Contrairement à la filière touristique (voir par la suite), celle-ci s'appuie sur une tradition d'apicueillette fortement ancrée au sein de la population locale. L'idée de promouvoir les pro-

5. Pour illustrer, le simple collecteur habitant un village avoisinant la forêt, gagne annuellement, en moyenne entre 4 et 17 euros par an, alors qu'un intermédiaire revendant les plantes dans l'agglomération de Tuléar peut gagner entre 280 euros et 480 euros par an (Raharinirina, 2004).

6. Raharinirina V. Enjeux, perspective et limites de la valorisation de la biodiversité à Madagascar : le cas de la forêt de Mikea. Programme EGER 1-UMR C3ED, Madagascar, mimeo, document de travail, 2004, $56 \mathrm{p}$. duits apicoles est généralement très bien appropriée par les acteurs locaux, dans une région qui regorge de plantes mellifères. Plusieurs projets ont été promus dans la zone d'étude. Le Programme de soutien au développement rural (PSDR) et l'Association nationale pour les actions environnementales (ANAE) financent des projets d'apiculture moderne. Regroupés en association de producteurs, les exploitants ont bénéficié d'aide conséquente comme l'indique le tableau 1.

Traditionnellement, la population a recours à l'api-cueillette. Cette pratique est très diffusée car elle ne nécessite aucun investissement et se pratique lors des activités de cueillette et de chasse. Cependant, le miel obtenu n'est pas homogène, contenant des débris de couvain. La production apicole de la région est de très faible qualité, aucune norme n'existe. Le rendement de l'exploitant est aussi très aléatoire parce que la taille des rayons varie d'un endroit à un autre, la production ainsi obtenue tient compte aussi de ce paramètre. Les enquêtes ont montré que le rendement moyen avoisine les 15 litres/an. Le revenu annuel des exploitants de produits apicoles de la région varie beaucoup selon le type d'exploitant. Un apiculteur traditionnel de la forêt ne gagne que $30 \%$ à $50 \%$ de ce que gagne un revendeur de miel à Tuléar?

Pour ce qui est de la structuration de la filière, la production apicole de la région

7. S'il s'agit d'un apiculteur traditionnel, commercialisant 10 seaux de miel par semaine, le revenu annuel varie entre 280 euros et 410 euros. Or, celui d'un revendeur de miel dans la ville de Tuléar, pour une même production, peut être 2 à 3 fois supérieur (Raharinirina, 2004, $c f$. note 6). 
est généralement vendue dans les marchés locaux. Des collecteurs venant de l'agglomération de Tuléar viennent également dans la région. Il peut s'agir de collecteurs professionnels qui sont souvent des karana habitant Tuléar ou bien de collecteurs occasionnels. Le circuit du produit est court ; les produits apicoles provenant généralement des cueilleurs. Ils sont vendus par ces derniers sur les marchés locaux, aux membres de leurs communautés ou bien collectés par des intermédiaires. En outre, l'acheminement des produits vers les consommateurs finaux peut se faire à travers les marchés locaux s'il s'agit de consommateurs ruraux, et à travers les intermédiaires s'il s'agit de consommateurs urbains.

Certains exploitants de cette zone pratiquent l'apiculture dite traditionnelle, c'est-àdire qu'ils utilisent des troncs d'arbres troués ou des morceaux de bois pour domestiquer les colonies d'abeilles. Toutefois, ce type d'exploitation est rare dans la forêt des Mikea car il exige déjà une certaine maîtrise des différentes phases des opérations.

\section{La filière tourisme}

L'activité touristique est la plus balbutiante des trois filières promues dans la zone, et ce malgré la présence du site balnéaire d'Ifaty-Mangily, au sud de la forêt. Composé d'une vingtaine d'hôtels, le site d'Ifaty-Mangily se place parmi les dix sites les plus visités de Madagascar. L'attrait du lagon de Tuléar à des fins touristiques accroît la pression foncière sur le front de mer ce qui se traduit par un déplacement des nouveaux investissements vers le Nord, dans les communes de la Fimami. La perspective de rentes issues de l'activité touristique, notamment par l'accroissement des recettes de la commune à travers la vignette touristique, incite les communes à développer cette activité. Toutefois, la plupart des projets touristiques émanent de particuliers étrangers qui proposent des activités orientées vers la mer; le lien avec la conservation de la forêt n'est donc pas significatif. Les hôteliers ne s'appuient guère sur les actifs spécifiques de la forêt dans la promotion de leurs activités. Par ailleurs, ces projets concernent uniquement les communes de la forêt des Mikea qui ont accès à la mer ; c'est-à-dire sur la partie Ouest de la forêt ; alors que les fronts pionniers sont sur la partie Est, soit environ à $30 \mathrm{~km}$. Enfin, les impacts en termes d'emplois ou de revenus directs et indirects, s'ils sont non négligeables à Ifaty-Mangily en raison du nombre important d'hôtels, sont assez faibles dans les communes de la Fimami. L'effet de substitution censé caractériser l'alternative écotourisme est absent à l'heure actuelle.

Une autre forme de tourisme concerne les touristes scientifiques qui sont attirés par la vingtaine d'espèces faunistiques dont le Tolohoranto (Uratelornis Chimaera), oiseau endémique à la région. Malheureusement, là encore, les effets sont moindres dans la mesure où les touristes demeurent dans les communes d'Ifaty-Mangily et vont dans la forêt à la journée. Il y a donc très peu de temps passé dans les villages de la forêt ; la vente de produits artisanaux, par exemple, est donc très limitée et se concentre sur la partie sud de la forêt, c'est-àdire la commune de Ankililoaka.

Au bout du compte, les trois filières proposées conjointement par les représentants de la Fimami et par les institutions les soutenant n'ont pas réussi à créer un dynamisme économique et institutionnel suffisant pour pallier à l'arrêt de la déforestation, même en tant qu'activités complémentaires. La figure 1 synthétise l'état actuel de ces filières.

\section{Quelles conditions pour une valorisation effective ?}

Le bilan très mitigé de la valorisation économique de la forêt des Mikea en vue de fournir une alternative à la déforestation est cependant riche d'enseignements. Parmi les différentes études filière réalisées, plusieurs contraintes ont été relevées. Elles constituent alors autant de perspectives pour permettre à la fois de parvenir à une valorisation plus effective et en même temps d'éviter la reproduction de telles difficultés. Les enseignements de nos études ont montré l'existence de 
Figure 1. L'organisation des filières de valorisation

(les flèches indiquent le sens de déplacement des produits/services de la forêt)

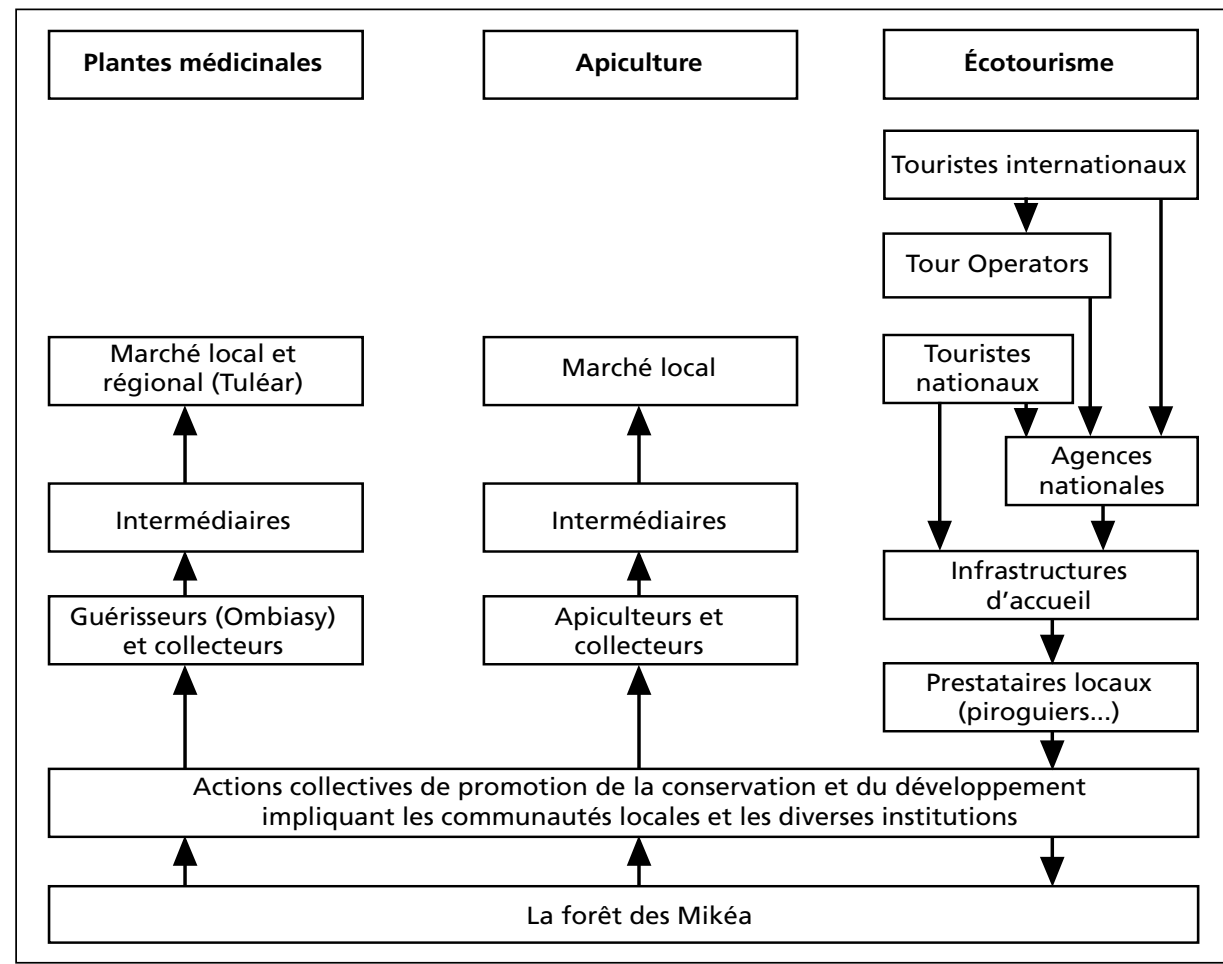

plusieurs contraintes et ce pour les trois filières avec toutefois une intensité diverse selon le type de projet.

Au plan de l'analyse économique, ces contraintes peuvent être regroupées en quatre catégories. Les deux premières se réfèrent aux nouvelles théories de développement local grâce notamment à « l'activation de ressources cognitives partagées » et à «l'ancrage territorial des activités ». Ces deux points forment les éléments primordiaux de ce que nous appellerions, à l'instar de Requier-Desjardins (2005), les conditions d'une orthogonalité des filières de biodiversité. Toutefois, l'application de ces théories dans le cas particulier de la gestion durable d'un écosystème fragilisé invite à tenir compte d'autres dimensions plus classiques en économie de l'environnement, à savoir la question des retombées économiques et celle de la durabilité écologique.

\section{L'activation de ressources cognitives partagées}

Les communes dans lesquelles sont promues les activités de valorisation sont celles qui bénéficient traditionnellement le plus de relais forts auprès des bailleurs de fonds et des services publics déconcentrés. Deux des trois communes étudiées ont été parmi les premières à élaborer leur schéma de planification communale, attirent depuis longtemps les institutions pour divers projets de développement ou d'infrastructures. L'existence d'un capital social important attire les bailleurs de fonds lorsque ceux-ci recherchent des zones pilotes pour la mise en place de projets spécifiques. C'est donc l'identification d'une capacité d'interactions entre acteurs institutionnels qui permet d'amorcer ces processus d'action collective.

Pour autant, l'activation de ces ressources cognitives n'est pas automatique ; c'est-à-dire que le seul partage d'un système de repré- 
sentation entre la Fimami et les institutions d'aide (ONG nationales et internationales ainsi que les bailleurs de fonds) ne suffit pas à rendre effective la filière de valorisation. L'enjeu consiste alors à inscrire le projet dans une dynamique de proximité qui combine proximité géographique et organisée (Gilly et Torre, 2000 ; Torre, 2004). Deux conditions permettent de faciliter et de favoriser l'interaction entre les parties prenantes et de contribuer ainsi à la mise en œuvre d'action collective de la part des acteurs situés sur un territoire donné :

- le partage d'un objectif commun sur la nature du projet,

- l'adéquation entre les pratiques traditionnelles et les nécessités techniques et organisationnelles (voire financières) de l'activité.

Ces deux conditions ne visent d'ailleurs pas forcément l'ensemble de la population ; il s'agit d'identifier au préalable les formes de pouvoir qu'exercent entre eux les différents acteurs. Comme le souligne Fauroux E. (2003), l'extériorité des projets et plus particulièrement l'absence de compréhension des structures micro-locales du pouvoir sont une des principales raisons de l'échec des projets.

L'exemple du tourisme est le plus symptomatique de ces contraintes. En effet, les promoteurs les plus actifs sont membres de la Fimami et/ou sont des acteurs économiques influents localement (mpanarivo). Ils fréquentent très souvent les centres urbains et côtoient ainsi les étrangers, les touristes et les acteurs nationaux des organismes de développement. Toutefois, la réticence d'une partie de la population quant au développement de toute forme d'activité touristique est forte. Plusieurs zones considérées comme sacrées et inaccessibles par les ainés (ray aman-dreny) sont proposées comme site de visite pour les touristes ce qui limite fortement la constitution de groupement ou d'associations locales promouvant le tourisme. De même, l'intrusion des étrangers (vazaha) dans certains endroits n'enthousiasme généralement pas la population locale. On constate donc un décalage très important entre les objectifs des projets touristiques et les modes de vie des populations concernées.

Ce décalage est renforcé par les contraintes imposées par ce type d'activité : les investissements en termes d'infrastructures, la pratique de langues étrangères, les conflits fonciers récurrents sont autant d'obstacles au développement de l'écotourisme. Les études menées dans le sud-ouest malgache, montre que très souvent le personnel qualifié impliqué dans l'activité touristique provient des grandes villes, Tuléar et la plupart du temps Antananarivo, la capitale. Même lorsque la gamme de service offerte est moyenne, lorsque la barrière de la langue n'est pas un problème, les prestataires de services sont d'anciens employés d'hôtel pour touristes étrangers et disposent alors d'un savoir-faire qui constitue une véritable barrière à l'entrée pour les autres acteurs.

L'absence évidente de proximité organisée autour de cette activité ne permet pas pour le moment d'aller au-delà de la simple proximité géographique liée à la présence du site balnéaire d'Ifaty-Mangily et qui demeure insuffisante dans le cadre d'une filière aussi longue et aussi exigeante (concurrence entre sites, normes de qualité, stabilité politique...).

Dans le cas de la filière plantes médicinales, la population locale joue le rôle de simples collecteurs individuels. Il n'existe pas de groupements. La filière plantes médicinales est en fait structurée en fonction des besoins des vendeurs sur le marché de Tuléar, ce qui explique en grande partie le rôle joué par les intermédiaires. Cette situation peut s'expliquer de deux manières.

- Premièrement, la difficulté de susciter une action collective à cause de l'objectif commun entre les acteurs de la filière. Des vendeurs de plantes médicinales des marchés de Tuléar, par exemple, viennent directement faire la collecte dans la forêt des Mikea, sans passer par les acteurs locaux ou les autorités dans cette zone. Face à cette absence d'articulation, ces derniers ne bénéficient donc pas de l'existence des ressources et de la 
détention de droits de propriété sur ces dernières. Il faut d'ailleurs noter que les plantes sont considérées par de nombreuses ethnies malgaches, notamment les masikoro principale ethnie de la région, comme un bien commun, accessible à tous. Par contre, en tant que ressources naturelles ayant des vertus thérapeutiques et médicinales, les plantes médicinales sont considérées par les communautés locales comme des dons de dieu (Zanahary) et ne doivent pas être vendues sur le marché pour générer des bénéfices. Il appartient au tradipraticien, détenant les connaissances traditionnelles de fournir les plantes avec leur utilisation thérapeutique à ceux qui sont dans le besoin.

- Deuxièmement, l'absence d'appui institutionnel et l'inexistence de promotion de la filière plantes médicinales par les organismes de développement rural travaillant dans la zone expliquent en grande partie l'absence de proximité organisée.

Quant à la filière apiculture, sa promotion dans la zone repose en grande partie sur l'utilisation de ruches langworth, sur la base de techniques dites modernes. Les groupements de paysans qui ont décidé de se professionnaliser dans l'apiculture moderne connaissent souvent des difficultés techniques et financières au moment du lancement de l'activité (désertion des essaims...). Ceci explique la faiblesse de la production et par conséquent des prévisions de vente. L'instabilité de la production pénalise fortement la filière à l'heure actuelle. Par conséquent, il s'avère difficile d'atteindre l'objectif commun fixé par les membres des groupements, à savoir l'écoulement de leurs produits sur les marchés local, national et international.

Cependant, contrairement aux deux autres activités, l'apiculture est un projet qui mobilise des ressources cognitives partagées par un nombre important d'acteurs. Les espoirs placés dans cette activité, comme d'ailleurs dans de nombreux autres endroits à Madagascar, se justifient a priori par une activation plus facile des ressources que dans le tourisme ou les plantes médicinales.

Globalement, l'action collective nécessite des modalités de coordination entre acteurs n'ayant pas toujours l'habitude de partager des valeurs et des objectifs communs. Les exemples de réussite de projets de valorisation ne reposent pas seulement sur l'implication des communautés locales mais également sur le partage de vue entre l'ensemble des parties prenantes (bailleurs de fonds, services déconcentrés de l'État, opérateurs privés...). Trop souvent malheureusement, l'accent est uniquement mis sur les organisations paysannes alors qu'il convient, dans un objectif de qualité, de prendre en compte l'ensemble des parties prenantes.

\section{L'ancrage territorial de l'activité}

Les conditions pour une meilleure valorisation reposent non seulement sur la coordination des acteurs mais également sur la compréhension des mécanismes marchands qui structurent l'organisation de la filière en question. En effet, les entretiens réalisés ont montré une difficulté transversale liée à une méconnaissance du fonctionnement de la filière.

La raison principale tient au fait que les institutions qui promeuvent ces stratégies de valorisation de la biodiversité partent d'une logique écologique pour aller vers une approche filière. Comme nous l'avons vu dans la première partie, la valorisation est vue comme un moyen permettant de limiter les effets négatifs de l'arrêt de la déforestation. Le cœur de l'initiative est avant tout la protection de la forêt. Par exemple, dans le cas des plantes médicinales, l'idée de la valorisation est née d'inventaires biologiques réalisés en 2000. C'est sur cette base que les potentiels d'exploitation de certaines essences ont été identifiés. Les études économiques ou financières sont rarement abordées. Ceci est d'autant plus vrai que les filières commerciales sont à créer comme dans notre zone d'étude.

Au-delà des questions relatives à l'action collective que nous avons abordée précédemment, il apparait évident que la plupart des difficultés rencontrées par les acteurs 
locaux dans le développement des activités de valorisation tiennent en grande partie au fait que les informations sur l'état de la filière et sur les spécificités de leur activité par rapport à d'autres ne sont pas disponibles localement. Aucune étude de marché, de réflexions sur la nature des débouchés, d'analyse des produits porteurs (quel type de miel ? mono ou poli floral ?) ou services spécifiques (quel site écotouristique valoriser? quel type de clientèle cibler?, etc.) n'ont été menées pour les trois filières.

Avoir connaissance du fonctionnement de la filière promue par le projet de valorisation est d'autant plus important qu'il ne s'agit pas d'orienter les populations locales vers des filières au sein desquelles elles n'auraient aucun pouvoir. Ce type de situation est notamment prévisible dans le cas des plantes médicinales. L'actif stratégique « connaissance en pharmacopée » est certes initialement aux mains des guérisseurs (ombiasy), ce qui leur confère un certain poids dans la filière ; cas des filières pilotées par l'amont (producer's driven). Toutefois, il est à craindre, en témoignent les débats sur la protection des savoirs et savoir-faire traditionnels dans le cadre des accords de bioprospection, que le maillon stratégique glissera irrémédiablement aux mains des firmes pharmaceutiques au fur et à mesure que le médicament (ou l'huile essentielle) sera mis sur le marché.

Il est alors important pour les organismes d'appui de bien identifier ce type de risques et d'orienter le cas échéant la promotion de cette activité sur des filières plus courtes au sein desquelles les populations locales garderont le contrôle de l'exploitation.

Bien que pilotées par l'aval (buyer's driven), les deux autres filières courent le même risque. Proposer du miel et des services touristiques parfaitement génériques, conduiraient les groupements à une absence totale de contrôle sur la filière ; en étant en concurrence avec d'autres produits (ou services) malgaches et a fortiori internationaux. Par exemple, les enquêtes menées auprès des touristes à Ifaty-Mangily montrent que la concurrence serait réelle avec les autres aires protégées Ranomafanana et Isalo qui sont intégrés aux circuits des tours operators internationaux et nationaux. La plupart des touristes présents dans la région ont visité ou planifient de le faire, ces deux aires protégées. L'intérêt de la visite de la forêt des Mikea reste problématique. De même, les essences mellifères de la forêt des Mikea conduisent à produire du miel poli floral, rendant le produit parfaitement générique au niveau de sa composition.

La seule option tangible est de relier ces produits et services à la forêt des Mikea, c'est-àdire à faire en sorte qu'ils constituent des « actifs spécifiques situés ». Il s'agirait de mettre en avant la typicité de ces produits et services en se référant aux particularismes de ce terroir (Requier-Desjardins, 2005), voire en mettant l'accent sur un ensemble de produits et services dans une logique de «paniers de biens » (Pecqueur, 2000). À l'image des écharpes en soie d'Arivonimamo ou des paniers en raphia de Brickaville, les projets peuvent mettre en avant les produits de la forêt des Mikea. Les membres actifs de la Fimami l'ont d'ailleurs bien compris : la présence « mystérieuse » des Mikea au sein de la forêt, présence médiatisée par les bailleurs de fonds, suscitent de la part de ces responsables locaux un intérêt pour le développement d'un tourisme dont l'actif spécifique se concentrait autour de la combinaison forêt (baobabs, flamants roses du lac de Voreha, lagon de Tuléar...) et population Mikea ${ }^{8}$. De ce point de

8. La présence de la population Mikea est source de controverses et fait l'objet des plus grandes interrogations quant à leur existence même... Le mystère qui entoure cette population est source de spécificité pour les tours operators malgaches. Par exemple, le site internet qui mentionne la population Mikea est celui de madatours, un des principaux sites de promotion touristique de Madagascar. Les Mikea y sont assimilés aux « curiosités de Madagascar». On peut y lire « dans la forêt sèche au nord de Tuléar vit un peuple mystérieux, retiré de la civilisation et isolé du reste du monde. Bel exemple de la réconciliation de l'homme avec la nature, les Mikea ont un mode de vie très particulier $»$ http://www.madatours.com/ curiosites/mikea/mikea_madagascar.htm . 
Graphique 1. Évolution comparée des bénéfices nets de l'exploitation du maïs et de l'apiculture moderne dans la forêt des Mikea (en Francs malgaches, année 2002)

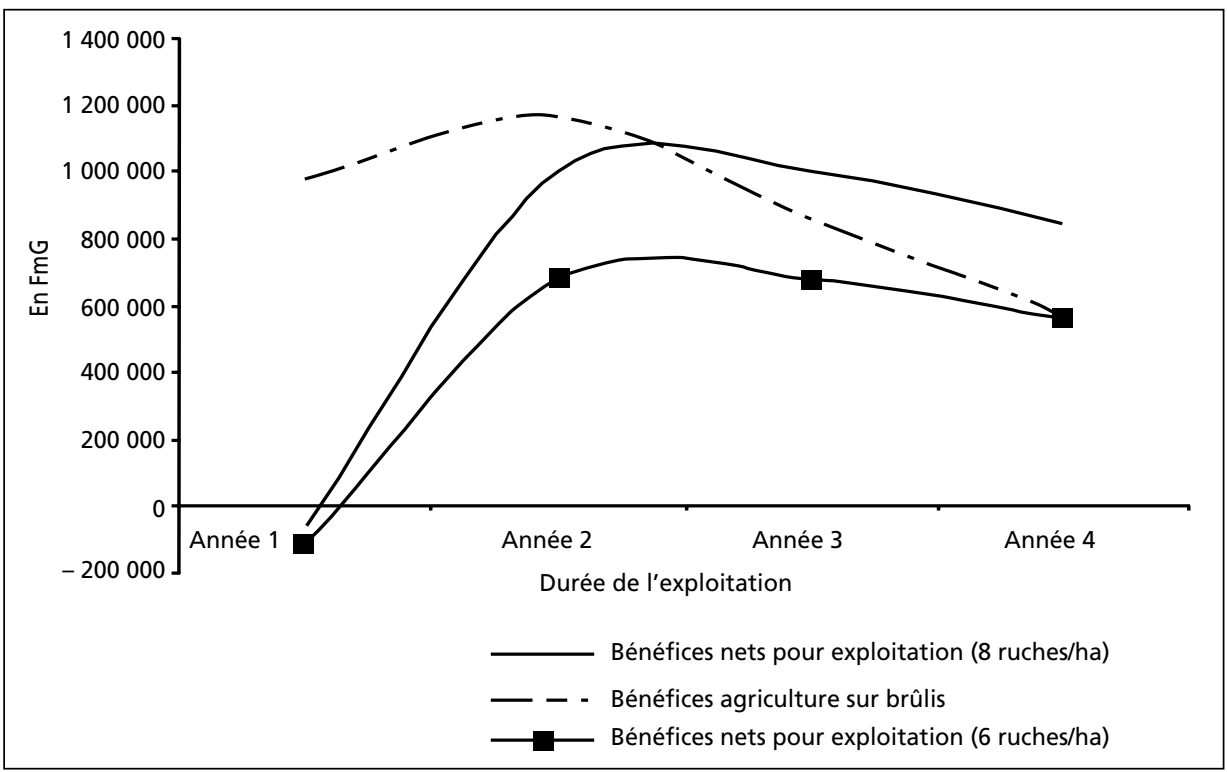

Sources : Méral et Razafimahatolotra (2002), Rakotosoa $(2004)^{9}$ et les calculs des auteurs

vue, l'activité serait complémentaire aux formes de tourisme développé à Ifaty-Mangily.

Les deux premiers facteurs, "activation des ressources cognitives » et « ancrage territorial des activités » constituent donc la clé de voute d'une bonne articulation entre filière et territoire en jouant sur les acteurs de la valorisation et sur les actifs valorisés. Toutefois, dans une économie paysanne telle qu'elle apparaît dans cette zone d'étude, deux autres conditions doivent également être prises en considération.

\section{Les impacts socio-économiques}

Si l'on adopte une démarche purement économique, il faut convenir que la réussite des projets de valorisation repose sur leur capacité à créer davantage de revenus que les exploitants perçoivent à travers la conversion de la forêt en terre agricole. Dans le cas présent, les évaluations économiques faites montrent que le bénéfice net retiré par un exploitant agricole pratiquant la culture du maïs sur brûlis peut atteindre la deuxième année un million de FMG/ha (environ $90 €$ )
(Méral et Razafimahatolotra, 2002). L'apiculture, seule activité pour laquelle il est possible de réaliser une simulation financière, conduirait à un bénéfice à l'hectare compris entre 48 et $80 € /$ ha/an dans le meilleur des cas (pas de désertion des colonies, bonne maîtrise des ruches de type langstroth) (graphique 1).

Par conséquent, même si la culture de maïs sur brûlis est une activité portant atteinte à la biodiversité, elle génère, à court terme, les deux ou trois premières années notamment, plus de revenus que les autres filières alternatives. Enfin, si l'on voulait poursuivre dans cette logique comptable, il faudrait compléter l'évaluation en prenant en compte le fait que les parcelles de forêt mises en culture fournissent à l'exploitant une quantité importante de bois (construction,

9. Rakotosoa N. Valorisation de la biodiversité par le miel : l'exemple de la forêt de Mikea. Programme EGER 1 - UMR C3ED, document de travail, Madagascar, 2004, $31 \mathrm{p}$. 
chauffe...) et une fois « abandonnées », elles subissent un processus de savanisation qui est très utile pour l'élevage, autre source de richesses (Razanaka et al, 2001). Il est en fait assez peu fréquent de trouver des projets de valorisation qui conduisent rapidement à créer des revenus supérieurs aux activités dommageables pour l'environnement. Ceci est d'autant plus fréquent que ces activités sont très rémunératrices comme cela est le cas avec la culture commerciale du maïs. Il est possible, comme nous l'avons vu précédemment, d'améliorer l'activité de valorisation à travers une approche filière en mettant l'accent sur l'accroissement des quantités produites comme le proposent Belcher et al. (2005) ou bien sur la proximité des centres urbains (Stoian, 2005).

Il est également possible d'envisager l'activité de valorisation sous l'angle des impacts socio-économiques et plus seulement sous l'angle des revenus des exploitants. Ainsi en adoptant une démarche plus socio-économique, c'est-à-dire allant au-delà de l'hypothèse de rationalité économique - dans le sens d'une prise de décision individuelle qui s'effectue sur la base d'un comparatif statique de coûts et de bénéfices privés - on constate qu'un critère de réussite des projets de la valorisation est lié à leur dimension communautaire. En effet, nous avons recensé trois formes de retombées économiques :

(i) la création de revenus directs ou induits pour la population locale ;

(ii) la création d'emplois ;

(iii) les retombées indirectes liées aux externalités positives engendrées par l'activité de valorisation.

Or dans des montages institutionnels de type privé, comme dans le tourisme par exemple, la création de revenus directs peut être importante mais les retombées au niveau local en termes d'emplois et/ou d'impacts indirects sont généralement faibles (Chaboud et al, 2004). De nombreuses études internationales montrent qu'à activités économiques équivalentes, les dispositifs de gestion communautaire ont la plupart du temps plus d'impacts que les dispositifs privés (Antinori et Gray, 2005 ; Jagger et al, 2005).

Plus encore, ces expériences montrent que les projets de valorisation doivent s'inscrire dans une démarche de développement local impliquant l'ensemble de la population des villages au sein desquels ces projets sont développés. Il s'agit alors de porter l'attention non pas sur une filière de valorisation seule mais sur une combinaison d'actions planifiées avec la population locale permettant à un grand nombre d'acteurs de bénéficier des retombées socio-économiques des projets de développement. Inscrire le projet de valorisation comme composante économique d'une stratégie de développement local peut permettre aux petits paysans d'orienter leurs activités vers des pratiques limitant la pression sur la forêt. Même si les revenus issus de chaque alternative sont limités et plus faibles que ceux issus de la déforestation, les paysans peuvent s'associer à ces initiatives si par ailleurs cela leur permet de ne plus avoir à établir des campements provisoires loin des villages pour pratiquer la déforestation, d'avoir accès à des points d'eau, à des écoles, à des dispensaires... Même si ces avantages sont de nature collective, contrairement aux revenus qui sont perçus au niveau du ménage, de nombreux exploitants des fronts pionniers sont prêts à orienter différemment leur pratique ; traduisant par là même une forme de rationalité qui va bien au-delà du simple calcul économique individuel. La valorisation économique apparait alors comme un moyen parmi d'autres d'accroître les avantages perçus par les membres du dispositif de gestion communautaire.

Bien évidemment, cette vision plus large des projets de valorisation économique est plus ambitieuse car, d'une part, elle fait appel à des processus de planification participative élaborée, longue, faisant intervenir de nombreux acteurs - ce qui est d'ailleurs le cas actuellement dans ce site d'étude - et, d'autre part, elle assujettit chaque projet à la réalisation effective des 
autres. Quoi qu'il en soit, les enquêtes qualitatives réalisées dans ces villages montrent que la faiblesse des revenus issus des projets de valorisation est certes mal vécue par les paysans impliqués, mais c'est surtout l'absence d'impacts socio-économiques significatifs qui les découragent.

\section{La limitation des effets contre-intuitifs}

Les études sur ces trois filières ont montré l'existence de risque d'effets pervers que nous décomposons en trois grands risques : le risque de surexploitation, le risque d'éviction d'acteurs ou d'usages traditionnels et enfin le risque de renforcement de la destruction plutôt que l'offre de véritables alternatives.

Le risque d'une surexploitation lié à des projets de valorisation peut survenir dans trois situations :

- premièrement, les perspectives de gains monétaires issus de la valorisation peuvent conduire à des dynamiques incontrôlées qui affectent les temps de régénérescence des écosystèmes valorisés. Ce cas de figure est très marqué dans le cas des plantes médicinales. Selon l'ONE, on note par ailleurs que cette situation est réelle pour le prunus africana et le raventsara, valorisés dans d'autres régions de Madagascar ;

- deuxièmement, le risque de surexploitation apparaît lorsque la valorisation a conduit des personnes peu habituées aux techniques de prélèvement par exemple à gaspiller la ressource. On observe cette situation dans le cas des plantes médicinales, lorsqu'une partie seulement de la plante est intéressante (écorce, tige, feuilles, etc.) et que pour des rentes de court terme, c'est la plante entière qui est prélevée ;

- troisièmement, la surexploitation apparaît de manière indirecte par exemple lorsque l'activité touristique accroît les prix des denrées localement (Chaboud et al, 2004). L'apparition de cette activité censée promouvoir la conservation de l'écosystème littoral conduit au contraire à une pression sur la ressource.
Le risque d'éviction d'acteurs ou d'usages traditionnels est perceptible dans la forêt des Mikea. Les sites touristiques proposés correspondent à des endroits vénérés par les populations locales. Les discussions autour des projets de développement sur le site d'Amboboka à Ankililoaka ont vu les anciens s'opposer fermement à toute utilisation d'un site sacré par des étrangers (vazahas)... De même, l'effet prix mentionné plus haut dans le cas des ressources halieutiques prisées par les touristes n'induit pas seulement un risque de surexploitation mais aussi de baisse de l'offre pour les populations locales qui ne peuvent « suivre» l'augmentation des prix. Ce risque existe également pour les autres produits valorisés. C'est le cas des plantes médicinales qui sont demandées sur le marché international et qui deviennent de véritables intrants pour des sociétés étrangères. Elles deviennent non seulement rares mais sont hors de portée pour les populations locales.

Le risque du rendorcement de la destruction concerne le caractère alternatif $d u$ projet, c'est-à-dire sa capacité à modifier les comportements des acteurs en vue de limiter la destruction de l'environnement. Le concept d'alternative qui sous-tend, dans la plupart des cas, la valorisation économique des ressources issues de la biodiversité repose sur le principe de choix entre plusieurs options. Or, les acteurs locaux ne décident pas de faire telle ou telle activité en fonction d'un calcul de type coûts-bénéfices, c'est-à-dire en choisissant l'activité qui rapporte le plus et en délaissant les autres. L'arbitrage se fera davantage sur la base de la combinaison permettant de limiter les risques de vulnérabilité pour les ménages les plus pauvres et d'obtenir un niveau de richesses (et/ou de pouvoir) le plus élevé possible pour les ménages les mieux dotés. Par conséquent, il est tout à fait envisageable de voir les projets de valorisation venir en complément des autres activités, même si elles se font concurrence à terme. 
DOSSIER

Ces trois types de risques montrent à quel point la pertinence supposée d'une filière n'implique en rien son efficacité en termes de durabilité de l'environnement. Il apparaît donc nécessaire d'associer les institutions porteuses du projet et les acteurs locaux dans le processus de valorisation le plus longtemps possible. Le tableau 2 présente les résultats des trois filières étudiées sous l'angle des conditions identifiés précédemment :

Tableau 2. Bilan de la situation des trois filières de valorisation

\begin{tabular}{|c|c|c|c|c|}
\hline \multicolumn{2}{|c|}{ Contraintes } & Filière touristique & Filière plantes médicinales & $\begin{array}{c}\text { Filière apiculture } \\
\text { (miel et autres produits) }\end{array}$ \\
\hline \multirow[b]{2}{*}{$\begin{array}{l}\text { Activation } \\
\text { des } \\
\text { ressources } \\
\text { cognitives } \\
\text { partagées }\end{array}$} & $\begin{array}{c}\text { Partage d'un } \\
\text { objectif commun } \\
\text { entre les différents } \\
\text { acteurs }\end{array}$ & Pour le moment inexistant & $\begin{array}{l}\text { Problème de communication } \\
\text { entre les tradi-praticiens et les } \\
\text { autres acteurs de la filière }\end{array}$ & $\begin{array}{l}\text { A priori en bonne voie si } \\
\text { l'organisme d'appui encadre sur } \\
\text { le long terme ce qui n'est pas le } \\
\text { cas aujourd'hui }\end{array}$ \\
\hline & $\begin{array}{c}\text { Adéquation avec } \\
\text { les pratiques } \\
\text { traditionnelles }\end{array}$ & \begin{tabular}{|c|} 
A priori problématique à court \\
terme : le tourisme n'est pas une \\
pratique habituelle et si la forme \\
de tourisme s'oriente vers un \\
tourisme plus de découverte \\
et/ou culturel, alors existence \\
d'un risque de conflit entre \\
acteurs sur les lieux de culte
\end{tabular} & $\begin{array}{c}\text { Adéquation tant que } \\
\text { le tradi-praticien conserve } \\
\text { la maîtrise de son savoir-faire }\end{array}$ & $\begin{array}{l}\text { Adéquation globale malgré } \\
\text { les problèmes liés } \\
\text { au passage d'une activité } \\
\text { api-cueillette à une apiculture } \\
\text { plus élaborée }\end{array}$ \\
\hline \multicolumn{2}{|c|}{$\begin{array}{l}\text { Ancrage territorial } \\
\text { de l'activité }\end{array}$} & $\begin{array}{l}\text { Risque d'absence de contrôle } \\
\text { de la part des acteurs locaux } \\
\text { dans une filière buyer's driven. } \\
\text { Typicité potentiellement forte }\end{array}$ & \begin{tabular}{|c|} 
Filière courte voire très courte. \\
Les opportunités sont sans doute \\
à trouver au niveau des marchés \\
de la ville de Tuléar au risque \\
sinon de perdre le maillon \\
stratégique (connaissance \\
pharmacopée) \\
\end{tabular} & $\begin{array}{l}\text { Filière courte pour le moment } \\
\text { et malgré des problèmes de } \\
\text { productions irrégulières } \\
\text { il existe un potentiel d'ancrage } \\
\text { territorial important de type } \\
\text { origine du produit, voire } \\
\text { commerce équitable }\end{array}$ \\
\hline \multicolumn{2}{|c|}{$\begin{array}{c}\text { Création de revenus et } \\
\text { impacts socio-économiques }\end{array}$} & \begin{tabular}{|c|} 
Les études réalisées sur d'autres \\
sites montrent que la rente \\
touristique échappe aux \\
communautés locales; \\
seuls des effets indirects \\
(vente d'artisanat) peuvent être \\
perceptibles. Privilégier le \\
tourisme communautaire à \\
vocation culturelle peut être une \\
option intéressante. Les impacts \\
peuvent être importants en \\
termes d'infrastructure \\
\end{tabular} & $\begin{array}{l}\text { La création de revenus peut être } \\
\text { importante mais mal répartie } \\
\text { (souvent, les acteurs ayant accès } \\
\text { au marché de Tuléar détiennent } \\
\text { et monopolisent la quasi-totalité } \\
\text { des bénéfices de la filière). } \\
\text { Les impacts sont faibles }\end{array}$ & $\begin{array}{l}\text { Des trois activités promues, } \\
\text { l'apiculture a le potentiel le plus } \\
\text { élevé de création de revenus } \\
\text { pour les populations } \\
\text { locales (graphique 1); son } \\
\text { organisation sous forme de } \\
\text { groupement permet également } \\
\text { une redistribution plus } \\
\text { importante. Pour le moment les } \\
\text { impacts sont faibles }\end{array}$ \\
\hline \multirow{3}{*}{$\begin{array}{l}\text { Effets } \\
\text { contre- } \\
\text { intuitifs }\end{array}$} & $\begin{array}{c}\text { Risque de } \\
\text { surexploitation }\end{array}$ & $\begin{array}{l}\text { Risque important si le nombre } \\
\text { de touriste est élevé } \\
\text { (déchets...) ; dans certains cas, } \\
\text { les prix pratiqués envers les } \\
\text { hôteliers sont plus élevés que } \\
\text { pour les populations locales }\end{array}$ & $\begin{array}{c}\text { Risque élevé si la valorisation } \\
\text { se fait en dehors des règles } \\
\text { traditionnelles et lorsqu'il s'agit } \\
\text { de plantes fortement demandées } \\
\text { sur le marché }\end{array}$ & Pas de risque \\
\hline & $\begin{array}{c}\text { Risque de } \\
\text { modification } \\
\text { et/ou d'éviction } \\
\text { des usages } \\
\text { traditionnels } \\
\end{array}$ & \begin{tabular}{|c|} 
Risque important observé sur le \\
site d'Amboboke (conflit entre \\
jeunes et anciens sur l'utilisation \\
d'un site de culte à des fins \\
touristiques)
\end{tabular} & Risque faible & $\begin{array}{c}\text { Pas de risque, I'apiculture même } \\
\text { moderne (ruches Langworth) } \\
\text { n'exclut pas l'api-cueillette }\end{array}$ \\
\hline & \begin{tabular}{c|} 
Risque de \\
complémentarité
\end{tabular} & \multicolumn{3}{|c|}{$\begin{array}{l}\text { A priori pas de risque pour les trois activités, mais tout dépend de la distance entre les lieux où se } \\
\text { déroule la valorisation et ceux où s'effectue la déforestation }\end{array}$} \\
\hline
\end{tabular}




\section{Conclusion}

En appréhendant la problématique de la valorisation sous l'angle territorial et non pas à partir d'une seule ressource, cet article a cherché à montrer les difficultés auxquelles sont confrontés les acteurs promouvant ce type d'initiative dans un territoire sujet à un dommage écologique particulier. La lenteur du processus de mise en œuvre de ces projets de valorisation qui découle de la complexité des modalités de gestion pluraliste de l'environnement, contraste avec l'urgence écologique, la pression des bailleurs de fonds et la situation de vulnérabilité dans laquelle se trouvent les paysans ayant accepté (temporairement ?) d'arrêter la déforestation.

Plusieurs pistes de réflexion ont été avancées. L'inscription des projets de valorisation dans une dynamique de planification locale et participative, la préférence pour des montages de type communautaire basée sur des actifs spécifiques situés et le déve- loppement de filières courtes au niveau régional sont les options qui apparaissent les plus susceptibles de rendre effectifs ces projets et d'éviter que les paysans ne perdent le contrôle de leur activité dans des filières globales. De même, la coordination entre acteurs (services de l'État, opérateurs privés, organisations paysannes...) apparaît comme une nécessité dont les contours ne sont pas définis a priori.

Quoi qu'il en soit, aux contraintes endogènes identifiées dans cet article s'ajoutent celles plus générales de sécurisation foncière et de fiscalité qui dépassent largement la seule problématique de la valorisation mais sans lesquelles ces activités génératrices de revenus ne seraient pas durables.

Les auteurs remercient Christine Aubry, Emmanuel Fauroux, Géraldine Froger, Denis RequierDesjardins et Christian Castellanet pour leurs commentaires. Les auteurs restent seuls responsables du contenu de ce texte.

\section{RÉFÉRENCES BIBLIOGRAPHIQUES}

Aknin A., Aubry C., Méral P. (2007). Risques et stratégies paysannes : le cas de la forêt des Mikea. In Chaboud C., Froger G., Méral P. (Eds), «Le développement durable dans les pays en développement : utopie ou nécessité ? Le cas de Madagascar », Karthala, à paraître.

Andriananja H., Raharinirina V. (2004). Institutions, acteurs et modalités de coordination : quels enjeux pour la durabilité et la gouvernance des ressources naturelles à Madagascar? Mondes en Développement, vol. 32, n 3, p. 73-87.

Antinori C., Gray D.-B.(2005). Community Forests Enterprises as Entrepreneurial Firms: Economic and Institutional Perspectives from Mexico. World Development, vol. 33, $\mathrm{n}^{\circ}$ 9, p. 1529-1543.

Aubry C., Ramaromisy A. (2003). Typolo- gies d'exploitations agricoles dans un village du front pionnier de la forêt des Mikea (Sud Ouest de Madagascar). Cahiers Agricultures, ${ }^{\circ} 12$, p. 153-165.

Belcher B., Ruiz-Pérez M., Achdiawan R. (2005). Global Patterns and Trends in the Use and Management of Commercial NTFPs: Implications for Livelihoods and Conservation. World Development, vol. 33, n 9, p. 1435-1452.

Blanc-Pamard C., Milleville P., Grouzis M., Lasry F. et Razanaka S. (2005). Une alliance de disciplines sur une question environnementale : la déforestation en forêt des Mikea (Sud-Ouest de Madagascar). Natures Sciences et Sociétés, $\mathrm{n}^{\circ} 13$, p. 7-20.

Chaboud C., Méral P., Andrianambinina A. (2004). Le modèle vertueux de l'écotou- 
risme : mythe ou réalité ? Mondes en Développement, vol. 32, $\mathrm{n}^{\circ} 125$, p. 11-32.

Fauroux E. (2003). Structures micro-locales du pouvoir et gouvernance dans les villages de l'Ouest malgache. In Langlois M., Méral P., Raharinirina Rabaovololona L. et Ralalaoherivony B.-S., (Dir.) « La gouvernance locale à Madagascar : représentation, modélisation, participation », Cahier du C3EDM, n 3, p. 2-18.

Fauroux S. (2000). Instabilité des cours du maïs et incertitude en milieu rural : le cas de la déforestation dans la région de Tuléar (Madagascar). Revue Tiers-Monde, $\mathrm{n}^{\circ} 164$, p. 815-839.

Fiéloux M., Lombard J. (1990). Élevage et société : étude des transformations socioéconomiques dans le Sud-Ouest malgache : l'exemple du couloir d'Antseva. Antananarivo, Orstom et ministère de la Recherche, Collection Aombe 1, 220 p. Froger G., Méral P., Herimandimby V. (2004). The Expansion of Governance in the Environmental Policies in Developing Countries: the example of Madagascar. International Journal for Sustainable Development, vol. 7, $\mathrm{n}^{\circ}$ 2, p. 164-184. Gilly J.-P., Torre A. (2000). Dynamiques de proximité. Paris, L'Harmattan, 301 p. Jagger P., Pender J., Gebremedhin B. (2005). Trading Off Environmental Sustainability for Empowerment and Income: Woodlot Devolution in Northern Ethiopia. World Development, vol, ${ }^{\circ}$ 9, p. 14911510.

Lagarde K., Rakotovelo N. (2004). Étude de la filière apiculture en vue du développement de l'exportation, réalisée pour le compte du FSP Gestion Décentralisée des Ressources Naturelles - SCAC Madagascar, CITE Antananarivo, rapport définitif, CD Rom.

Méral P., Razafimahatolotra D. (2002). Les dynamiques économiques liées à la déforestation de la forêt des Mikea : quelques enseignements. Communication au «Centenaire de l'Académie Nationale des
Arts, des Lettres et des Sciences de Madagascar », le 3 octobre 2002, Fianarantsoa, Madagascar.

Méral P., Raharinirina-Douguet V. (2006). En attendant les zébus... les enjeux de la gestion durable de la forêt des Mikea. Études rurales, $\mathrm{n}^{\circ} 178$, juillet-décembre 2006, p. 161-180.

Minten B., Méral P. (2006). Commerce et environnement : impacts de l'exportation de maïs sur les forêts d'épineux du SudOuest malgache. Rapport pour le programme Trade Liberalization, Rural Poverty and the Environment, WWF et Banque mondiale.

Pecqueur B. (2000). Qualité et développement territorial : l'hypothèse du panier de biens territorialisés. Économie Rurale, $n^{\circ} 261$, janvier-février, p. 37-49.

Razanaka S., Grouzis M., Milleville P., Moizo B., Aubry C. (2001). Sociétés paysannes, transitions agraires et dynamiques écologiques dans le Sud-Ouest de Madagascar. Antananarivo, CNRE/IRD, $400 \mathrm{p}$.

Requier-Desjardins D. (2005). La valorisation économique de la biodiversité : ancrage territorial et gouvernance des filières. In Reveret J.-P. et Weber J. (Eds), «Économie de l'environnement et des ressources naturelles », Liaison ÉnergieFrancophonie, numéro spécial 66-67, p.77-81.

Sunderland T., Ndoye O. (2004). Forest Products, Livelihoods and Conservation : Case Studies of Non-Timber Forest Product Systems - Volume 2 - Africa. Center for International Forestry Research, $333 \mathrm{p}$.

Stoian D. (2005). Making the Best of Two Worlds: Rural and Peri-Urban Livelihoods Options Sustained by Nontimber Forest Products from the Bolivian Amazon. World Development, vol, ${ }^{\circ}$ 9, p. 14731490.

Torre A. (2004). Proximité et territoires. Économie rurale, 2004, $\mathrm{n}^{\circ} 280$, p. 2-7. 